\title{
Physical conditions of star forming sites in the S247/252 molecular complex
}

\author{
C . Koempe ${ }^{1,2}$, G. Joncas ${ }^{3}$, J.G.A. Wouterloot ${ }^{4}$, and H. Meyerdierks ${ }^{5}$ \\ ${ }^{1}$ IRAM, Granada, Spain \\ ${ }^{2}$ Max-Planck-Institut für Radioastronomie, Bonn, Fed. Rep. of Germany \\ ${ }^{3}$ Dépt. de Physique, Université Laval, Québec, Canada \\ ${ }^{4}$ I. Physikalisches Institut, Universität zu Köln, Köln, Fed. Rep. of Germany \\ ${ }^{5}$ Radioastronomisches Institut, Universität Bonn, Bonn, Fed. Rep. of Germany
}

\section{Introduction}

By now, it is well established that massive stars form in giant molecular clouds. Numerous studies have shown that star formation, instead of being spread uniformly throughout molecular clouds, occurs in dense condensations located within these clouds. The physical conditions in these condensations are therefore critical input parameters for any theory of star formation.

In the following, we present the results of an ongoing case study of the molecular cloud complex associated with the HII regions S247 and S252. The fundamental idea is to completely map the molecular complex with low spatial resolution $\left(\sim 4^{\prime}\right)$ in a ${ }^{13} \mathrm{CO}$ line, then identify likely sites of recent star formation, and finally study these sites with high spatial resolution. This approach reveals the distribution of star forming sites throughout the molecular cloud and their physical conditions. The comparison of several studies of this kind will hopefully improve our understanding of the star formation process in galactic molecular clouds.

\section{Observations}

We used the $2.5 \mathrm{~m}$ antenna of the Observatoire de Bordeaux to map the molecular complex S247/252 (distance $2.2 \mathrm{kpc}$ ) completely in the ${ }^{13} \mathrm{CO}(\mathrm{J}=1-0)$ line. Additional ${ }^{12} \mathrm{CO}(\mathrm{J}=1-0), \mathrm{CS}(\mathrm{J}=2-1)$ and $\mathrm{HCO}^{+}(\mathrm{J}=1-0)$ data were obtained towards S247. A search for $\mathrm{OH}$ and $\mathrm{H}_{2} \mathrm{O}$ masers was carried out using the quasi-meridian radiotelescope at Nançay, France, and the $100 \mathrm{~m}$ antenna at Effelsberg, Germany, respectively. We also obtained higher resolution (40") data towards the four IRAS point sources $06061+2151,06058+2138,0607+2138$, and $06069+2142$ located in the S247 molecular cloud in the $\mathrm{NH}_{3}(1,1)$ and $(2,2)$ lines using the Effelsberg antenna. The first two sources were mapped, while the line intensities of the other two sources were only measured towards their central position.

\section{Data analysis}

In the ${ }^{13} \mathrm{CO}(\mathrm{J}=1-0)$ line, two main molecular clouds are seen: a northern cloud close to the $\mathrm{S} 247$ HII region, and a southern cloud associated with the S252 optical nebula. They appear to be linked by a bridge of molecular gas. Several fragments can be distinguished in the S247 and the S252 cloud. Towards S247, the same fragments are seen in our CS and $\mathrm{HCO}^{+}$data indicating a minimum $\mathrm{H}_{2}$ density of $10^{4} \mathrm{~cm}^{-3}$. The same spatial structure as that seen in the ${ }^{13} \mathrm{CO}$ data is revealed by the IRAS data. Combining our ${ }^{13} \mathrm{CO}$ and ${ }^{12} \mathrm{CO}$ data, we found the total mass of the S247 cloud to be $37,000 \mathrm{M}_{\odot}$ which is similar to that of the $\mathrm{S} 252$ cloud $\left(25,000 \mathrm{M}_{\odot}\right)$ determined by Lada and Wooden (1979). We searched the IRAS point source catalog for objects that are located inside the ${ }^{13} \mathrm{CO}$ boundary of the molecular complex: 47 sources were found. Those sources that are located close to high density fragments are likely to be young stellar objects. Water masers were 
found towards five IRAS sources, and two OH masers in the vicinity of the S247 optical nebula. A detailed account of our mm, IR, and maser emission data can be found in Koempe et al. (1989). Contour maps of $\mathrm{NH}_{3}(1,1)$ obtained towards IRAS $06061+2151$ and IRAS $06058+2138$, displayed in Fig. 1, show a maximum close to the position of the IR source. The kinetic temperatures $\mathrm{T}_{K}$ derived from the ammonia data are also shown. In both cases, the maximum temperature is found close to the position of the IR source but decreases fast as one moves away from the source. This behavior is expected if the IR source is heating the surrounding gas from the inside. The kinetic temperatures derived for IRAS $0607+2138$ and IRAS $06069+2142$ are $18 \mathrm{~K}$ and $24 \mathrm{~K}$, respectively. A minimum $\mathrm{H}_{2}$ density of $10^{4} \mathrm{~cm}^{-3}$ was derived towards all four IR sources on the basis of the $\mathrm{NH}_{3}$ observations.

\section{Conclusion}

The S247/252 molecular complex is composed of two well separated clouds that are linked by a bridge of molecular material. Both clouds show a number of fragments. It should be noted that the masses of these fragments lie in the range of small molecular clouds. In the S247 cloud each fragment is associated with at least one IRAS point source. Towards five IR sources, $\mathrm{H}_{2} \mathrm{O}$ masers were detected indicating that these sources are indeed young stellar objects. Four IR sources are associated with compact HII regions (S252 A, S252 B, S252 C, and S252 E). It is thus likely that the IRAS sources, distributed throughout the S247/S252 gas complex, represent young stellar objects at different evolutionary stages. The mechanism(s) that triggered star formation in this particular complex remain(s) still to be discovered.

\section{References}

Koempe C., Joncas, G., Baudry, A., Wouterloot, J.G.A., Astron. Astrophys., 221, (1989)

Lada, C.L., Wooden, D., Astrophys. J., 232, 158, (1979)

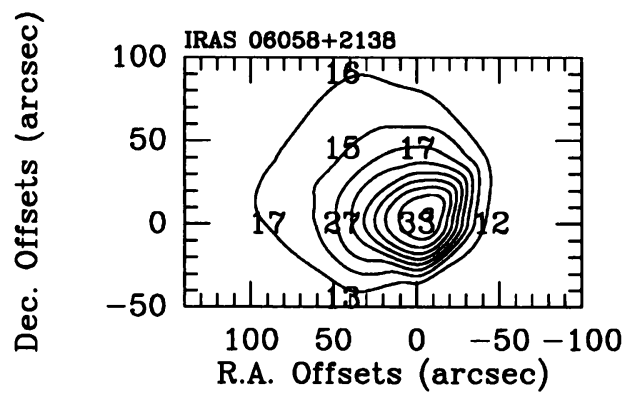

Fig. 1a

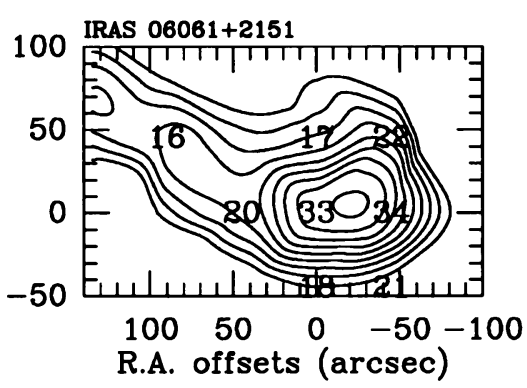

Fig. 1b

Fig. 1a: Integrated $\mathrm{NH}_{3}(1,1)$ contour plot of IRAS $06058+2138$. The lowest contour level is $0.6 \mathrm{~K} \mathrm{~km} \mathrm{~s}^{-1}$ and the spacing between levels is $0.2 \mathrm{~K} \mathrm{~km} \mathrm{~s}^{-1}$.

Fig. 1b: Integrated $\mathrm{NH}_{3}(1,1)$ contour plot of IRAS $06061+2151$. The lowest contour level is $0.5 \mathrm{~K} \mathrm{~km} \mathrm{~s}^{-1}$ and the spacing between levels is $0.15 \mathrm{~K} \mathrm{~km} \mathrm{~s}^{-1}$.

In both maps, the IR source is located at the $(0,0)$ position. The bold face numbers give the kinetic temperature at different offset positions. 